\title{
Assessing the painful, uninflamed eye in primary care
}

\author{
Lucia Kuffova senior clinical lecturer, University of Aberdeen; honorary consultant ophthalmologist, \\ NHS Grampian ${ }^{1}$, John V Forrester emeritus professor, University of Aberdeen; clinical professor, \\ University of Western Australia ${ }^{2}$, Andrew Dick professor of ophthalmology ${ }^{3}$
}

${ }^{1}$ Section of Immunity, Infection and Inflammation, Division of Applied Medicine, University of Aberdeen, School of Medicine and Dentistry, Aberdeen AB25 2ZD, UK; ${ }^{2}$ Ocular Immunology Program, Centre for Ophthalmology and Visual Science, University of Western Australia, Western Australia, 6009 Australia; and Centre for Experimental Immunology, Lions Eye Institute, Nedlands, WA 6009, Australia; ${ }^{3}$ Academic Unit of Ophthalmology, School of Clinical Sciences, University of Bristol, Bristol BS8 1TD; National Institute for Health Research (NIHR) Biomedical Research Centre at Moorfields Eye Hospital and University College London Institute of Ophthalmology, London EC1V 2PD, UK

Eye pain is common. Around 2-5\% of general practice patients have an eye complaint featuring pain in and around the eye (ocular, periocular, and periorbital pain, described here collectively as eye pain). ${ }^{1}$ Among patients attending an ophthalmic emergency department in New Zealand, more than $50 \%$ of new referrals were from general practices, ${ }^{2}$ with eye pain being the most common symptom. The authors suggested that referrals could be reduced with better initial diagnosis.

Eye pain with an inflamed, red eye is common and can be readily triaged for a management plan, in most cases by simple examination with a direct ophthalmoscope ${ }^{3}$ or pen torch. This is discussed elsewhere in relation to the differential diagnosis and treatment of conjunctivitis ${ }^{4}$ and when to refer for slit-lamp examination for sight threatening ocular surface inflammation or uveitis. ${ }^{5}$ Eye pain in an uninflamed (white) eye is less common. ${ }^{6}$

Guidelines for the diagnosis of conjunctivitis have been produced by the Dutch College of General Practitioners, ${ }^{7}$ and evidence based summaries on management of the red eye are available (http://cks.nice.org.uk/red-eye), but no validated guidelines for the painful, inflamed or uninflamed, eye have been developed. The present advice is based on a review of the literature (see box 1 for details) and a questionnaire based survey of patients attending an emergency hospital eye service (box 2).

The painful uninflamed eye, particularly if associated with reduced vision, presents a difficult diagnostic challenge, but key causes of the pain can be quickly deduced by using the steps below.

\section{What does the patient with eye pain fear most?}

Patients with eye pain are most often concerned about a possible threat to sight, as shown by our recent survey of patients attending hospital eye services (see box 2). Observational studies and the results of a controlled study ${ }^{10}$ also reported that patients have difficulty describing the pain in terms of its onset, duration, nature, and severity. A systematic review of therapies for trigeminal neuralgia revealed that, if the patient feels there is a low or negligible risk to sight, relief of pain becomes the predominant concern. ${ }^{11}$

\section{Initial assessment}

When a patient presents with eye pain in an otherwise "quiet" uninflamed eye, it is critical to identify potential sight threatening conditions with the following steps:

1. Ask if the pain is in or around the eye

2. Ask if the vision has changed

3. Check visual acuity (such as Snellen chart testing)

4. Check if the apparently white eye has any signs of inflammation even if mild: examine carefully the external eye, including those parts of the globe under the lids, for signs of occult trauma (subconjunctival haemorrhage), conjunctivitis, or foreign bodies (see below and fig $1 \Downarrow$ ).

Both pain located in the eye (ophthalmic eye pain) and pain located around the eye (non-ophthalmic eye pain) are relatively common. ${ }^{2}$ In most cases the threat to sight is low (even in inflamed eyes). However, determining if this risk exists is the main object of the consultation, and requires further steps in the assessment, which should address all symptoms not only the pain. 


\section{The bottom line}

- For the patient with a painful, uninflamed eye, it is crucial to check vision—reduced visual acuity is the major alarm signal of serious eye disease

- Refer all patients with eye pain who have reduced vision

- Eye pain without visual loss or redness is most often due to non-sight threatening conditions. A careful history and simple, direct examination will differentiate serious from less serious conditions

- Be alert to potentially sight threatening or life threatening conditions (such as giant cell arteritis, intracranial aneurysm, optic neuritis, and raised intracranial pressure).

- Be alert to occult eye disease (such as chronic glaucoma, chronic uveitis, and unsuspected trauma)

\section{Box 1: Methods of literature search}

We conducted a literature search using PubMed Medline from January 1970 to May 2014. We restricted our data collection to papers with the primary descriptors of "eye pain," "eye strain," or "eye discomfort," avoiding those with the primary descriptor "red eye" or "visual loss." We identified several systematic reviews, guidelines, or educational papers, ${ }^{45}$ and observational reports based on expert opinion, but no randomised controlled trials on eye pain in the uninflamed eye. These were generally on specific conditions such as Sjögren's syndrome, dry eye disease, migraine, trigeminal neuralgia, atypical facial pain, and the ocular ischaemic syndrome, ${ }^{9}$ as well as side effects of drugs and conditions such as eye strain. ${ }^{10}$

\section{Box 2: Details of questionnaire survey of patients at an emergency hospital eye service}

We conducted a questionnaire based survey of consecutive patients attending two UK ophthalmic emergency units over a two week period (Aberdeen Royal Infirmary and Bristol Eye Hospital, unpublished data). Patients with eye pain were asked for more detail on the pain; whether they had lost vision; whether the eye had become red; and their main concern regarding these symptoms (response rate $43 \%$ of 543 patients).

Most patients had both ocular pain and redness. Eye pain was located to the eye itself in slightly less than half the patients, while just over half complained of pain around or behind the eye. More patients were concerned about risk of losing vision (58\%) than the pain or redness. Deep severe pain and eye pain in both eyes were uncommon.

\section{What to look for next \\ Ophthalmic causes of eye pain}

Serious sight threatening causes of eye pain in the uninflamed eye in which the vision may or may not be reduced at the time of examination include occult trauma, posterior uveitis (fig $2 \Downarrow$ ), optic neuritis (fig $3 \Downarrow$ ), and, rarely, chronic glaucoma (fig $4 \Downarrow$ ) (see table $1 \Downarrow$ ).

In checking for these conditions, ask:

- Is there a history of recent or past trauma? This includes pain after accidental or surgical trauma (pain after cataract and laser refractive surgery may rarely persist for weeks to months)

- Is there evidence of trauma on examination of the external eye and lids (for example, in the pupil shape and reactions to light or colour of the iris)? Examine with a bright pen torch and a direct ophthalmoscope, include the region of the globe under the eyelid (fig $1 b \Downarrow$ ). Evert the upper lid with a cotton bud (a useful check for foreign bodies, fig $1 \mathrm{c} \Downarrow$ ). A retained subtarsal, periocular, or corneal foreign body can cause pain in an uninflamed eye. A retained intraocular foreign body and intraocular haemorrhage due to blunt trauma may alter the colour of the iris (heterochromia)

- Is there low level eye "ache" with unexplained reduced vision (reduction of more than 2 lines on a Snellen chart)? This may suggest chronic uveitis (fig $2 \Downarrow$ ), scleritis (fig $5 \Downarrow$ ), or chronic glaucoma (fig $4 \Downarrow$ )

- Is the pain of a dull, persistent, occasionally throbbing nature with headache? This suggests chronic angle closure glaucoma or rubeotic (neovascular) glaucoma (fig $4 \Downarrow$ ), for instance, associated with retinal vein occlusion or diabetic eye disease. Angle closure may occur in patients with chronic obstructive pulmonary disease
- Is the pain of sudden onset or acute, especially on eye movement, and associated with visual loss? This set of symptoms is pathognomic of optic neuritis (fig $3 \Downarrow$ ).

Non-sight threatening ophthalmic causes of eye pain are the most likely to present to general practice. ${ }^{2}$ These include optical causes (refractive error, eye strain), ocular surface disease (dry eye disease, Sjögren's syndrome), and a range of lid diseases (fig $6 \Downarrow$ ) (see table $1 \Downarrow$ ). Careful examination, using a bright pen torch or a direct ophthalmoscope, of the globe, the external eye and the everted lids (using a cotton bud, see fig $1 \Downarrow$ ) will reveal the cause.

\section{Non-ophthalmic causes of eye pain}

Serious, sight threatening, non-ophthalmic causes of eye pain in the uninflamed eye, in which the vision may or may not be reduced at the time of examination, include various forms of vascular disease such as intracranial aneurysms, giant cell arteritis (more usually scalp pain), and, rarely, transient ischaemic attacks (see table $2 \Downarrow$ ).

In checking for these conditions, ask the patient:

- Is there a history or evidence of scalp pain or tenderness or jaw claudication or pain on swallowing, all features of giant cell arteritis?

- Is there a history of headache, which might indicate raised intracranial pressure, as well as less serious causes such as sinusitis, migraine, cluster headaches?

- Is there a history of diplopia or signs of ocular muscle paresis—such as lid droop, divergent squint—as can occur with impending rupture of an intracranial aneurysm?

- Is there a history of transient ischaemic attacks (episodes of unilateral severe visual loss, with pain or headache in the pre-recovery or post-recovery phase)? Transient ischaemic attacks are a rare cause of sight threatening eye pain and may predicate a stroke, particularly if they occur in younger adults ${ }^{12} 13$ 
- Is there a history of shingles or a vesicular rash suggesting shingles, leading to post-herpetic neuralgia? Periocular skin pain and tenderness may also occur before the onset of vesicles.

Non-sight threatening non-ophthalmic causes of eye pain are a common cause of eye pain presented in general practice and include conditions such as sinus disease, migraine, trigeminal neuralgia, a range of lid diseases, and unusual causes of atypical facial pain. ${ }^{14}$

\section{When to refer}

Patients with eye pain in an uninflamed eye should be referred to secondary care as follows:

- Immediately (within hours) - if there is evidence of acute onset sight loss (more than two lines on Snellen chart) or suspicion of giant cell arteritis or raised intracranial pressure

- Soon (within days) — if there is a perceived risk of sight loss based on the suspected diagnosis (such as chronic glaucoma or uveitis)

- For investigation - if there is no perceived threat to sight but the symptoms are affecting quality of life and wellbeing (such as cluster headaches, migraine, unmanageable ocular surface disease).

We acknowledge the important contributions of Professor Amada J Lee, University of Aberdeen, Division of Statistics, for assistance with analysing the data from the patient survey. We thank Kamran Khan, Oliver Chadwick, and Paul Chua, trainee ophthalmologists, NHS Grampian, for providing the clinical images.

Contributors: LK contributed to the design of the study, the survey of patients, and writing the paper. JVF contributed to the design of the study and writing the paper. ADD contributed to the design of the study, the survey of the patients, and writing the paper. JVF is guarantor for the paper.

Competing interests: We have read and understood the BMJ policy on declaration of interests and have no relevant interests to declare.

The views expressed are those of the authors and not necessarily those of the National Health Service, the NIHR or the Department of Health.

Provenance and peer review: Commissioned; externally peer reviewed.

1 Davey C, Hurwitz B. Red or uncomfortable eye. Occas Pap R Coll Gen Pract 1992;58:56-61.

2 Perumal D, Niederer R, Raynel S, McGhee CN. Patterns of ophthalmic referral and emergency presentations to an acute tertiary eye service in New Zealand. N Z Med J 2011;124:35-47.

3 Anderson DF, Sullivan PM, Luff AJ, Elkington AR. Direct ophthalmoscopy versus slit lamp biomicroscopy in diagnosis of the acute red eye. $J R$ Soc Med 1998:91:127-8.

4 Granet D. Allergic rhinoconjunctivitis and differential diagnosis of the red eye. Allergy Asthma Proc 2008;29:565-74.

5 Guly CM, Forrester JV. Investigation and management of uveitis. BMJ 2010;341:c4976.

6 Brazis PW, Lee AG, Stewart M, Capobianco D. Clinical review: the differential diagnosis of pain in the quiet eye. Neurologist 2002;8:82-100.

7 Rietveld RP, ter Riet G, Bindels PJ, Schellevis FG, van Weert HC. Do general practitioners adhere to the guideline on infectious conjunctivitis? Results of the Second Dutch National Survey of General Practice. BMC Fam Pract 2007;8:54.

8 Ramos-Casals M, Tzioufas AG, Stone JH, Siso A, Bosch X. Treatment of primary Sjogren syndrome: a systematic review. JAMA 2010;304:452-60.

9 Terelak-Borys B, Skonieczna K, Grabska-Liberek I. Ocular ischemic syndrome-a systematic review. Med Sci Monit 2012;18:RA138-144.

10 Vincent AJ, Spierings EL, Messinger HB. A controlled study of visual symptoms and eye strain factors in chronic headache. Headache 1989;29:523-7.

11 Zakrzewska JM, Linskey ME. Trigeminal neuralgia. BMJ Clin Evid 2009;2009:1207.

12 Saarela M, Sundararajan S, Strbian D. Young patient with headache and amaurosis fugax. Stroke 2014;45:e3-4.

13 Schelfaut D, Dhondt E, De Raedt S, Nieboer K, Hubloue I. Carotid artery dissection: three cases and a review of the literature. Eur J Emerg Med 2012;19:181-7.

14 Piagkou M, Demesticha T, Troupis T, Vlasis K, Skandalakis P, Makri A, et al. The pterygopalatine ganglion and its role in various pain syndromes: from anatomy to clinical practice. Pain Pract 2012;12:399-412.

15 Pareja JA, Cuadrado ML, Porta-Etessam J, Fernandez-de-las-Penas C, Gili P, Caminero $A B$, et al. Idiopathic ophthalmodynia and idiopathic rhinalgia: two topographic facial pain syndromes. Headache 2010;50:1286-95.

Cite this as: BMJ 2015;351:h3216

๑ BMJ Publishing Group Ltd 2015 


\section{Tables}

Table 1/ Ophthalmic causes of eye pain in an uninflamed (white) eye

\begin{tabular}{lll} 
Condition & Possible clinical indicator & Age group \\
Sight threatening & & Any \\
\hline Trauma & History & Any \\
\hline Posterior uveitis & Floaters & Adults \\
\hline Optic neuritis & Pain on eye movement & Adults \\
\hline Chronic glaucoma & Persistent dull headache & Adults \\
\hline Non-sight threatening & & Adults \\
\hline Refractive error & "Eye strain" & Adults \\
\hline Dry eye disease & "Grittiness" & Adults \\
\hline Sjögren's syndrome & Dry eyes and mouth & Older adults $(>65$ years) \\
\hline Meibomitis & Epiphora & Older adults $(>65$ years) \\
\hline Trichiasis & Entropion & Any
\end{tabular}

*A subtarsal foreign body (FB) may be completely symptomless, but it commonly induces an "FB" sensation which is more than a feeling of grittiness, rather a roughness located to a specific position under the lid. 
Table 2| Non-ophthalmic causes of eye pain in an uninflamed (white) eye

\section{Sight threatening}

\begin{tabular}{lll}
\hline Intracranial aneurysm & Third nerve palsy & Adults \\
\hline Benign intracranial hypertension (raised intracranial pressure) & Disc swelling & Adults \\
\hline Giant cell arteritis & Skin or scalp tenderness & Older adults (>65 years) \\
\hline Herpes zoster & Post herpetic neuralgia & Adults \\
\hline Herpes zoster & Pre-vesicle stage & Adults \\
\hline Non-sight threatening & \multicolumn{2}{l}{} \\
\hline Sinusitis & Chronic headache, retro-orbital pain & Adults \\
\hline Migraine & Headache, photophobia, nausea, vomiting & Any \\
\hline Trigeminal neuralgia & Severe episodic pain & Adults \\
\hline Atypical facial pain & Cluster headaches & Any \\
\hline
\end{tabular}

${ }^{*}$ In this condition the pain is restricted to the trigeminal nerve distribution. 


\section{Figures}
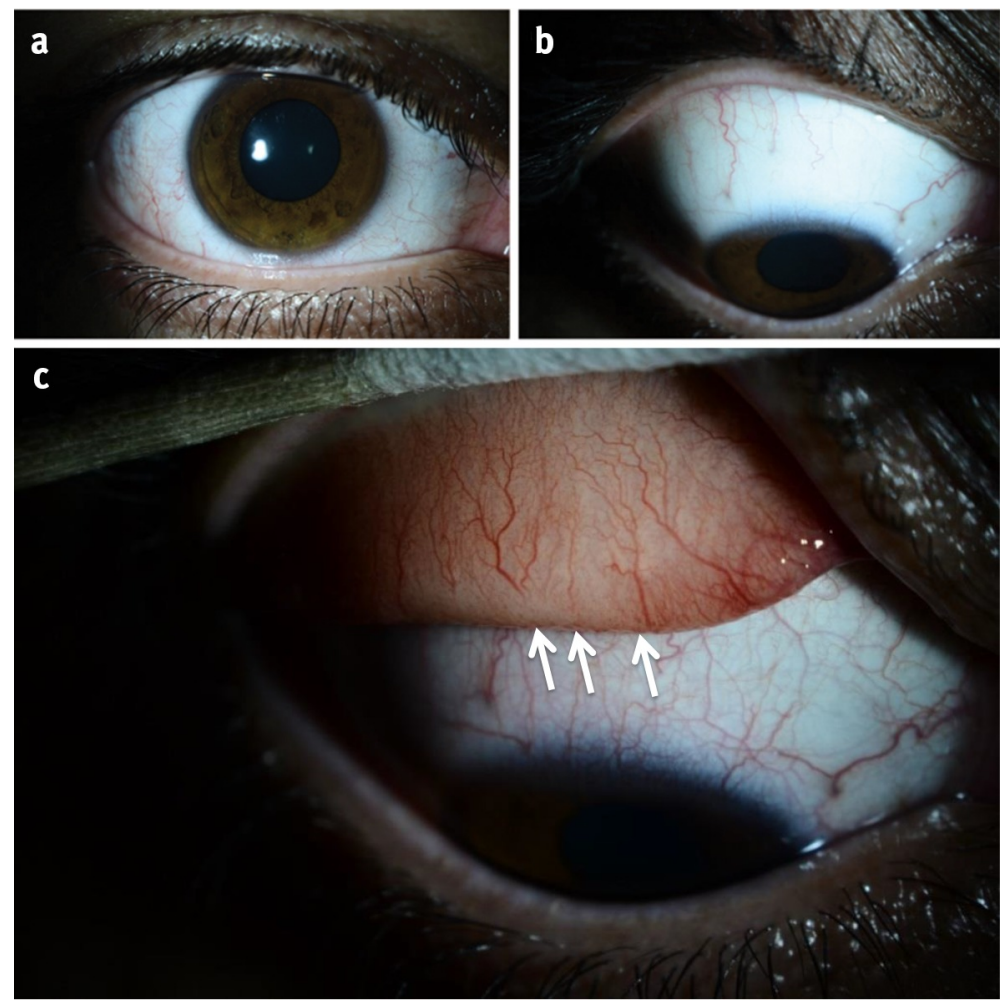

Fig 1 An apparently uninflamed white eye when in primary position of gaze (a) and looking down with eyelid retracted (b) masks a low level of inflammation caused by allergic papillary conjunctivitis and revealed by everting the upper lid (c): note the papillae made visible at margin by everting the lid (arrows) and the prominent conjunctival vessels on the everted lid surface

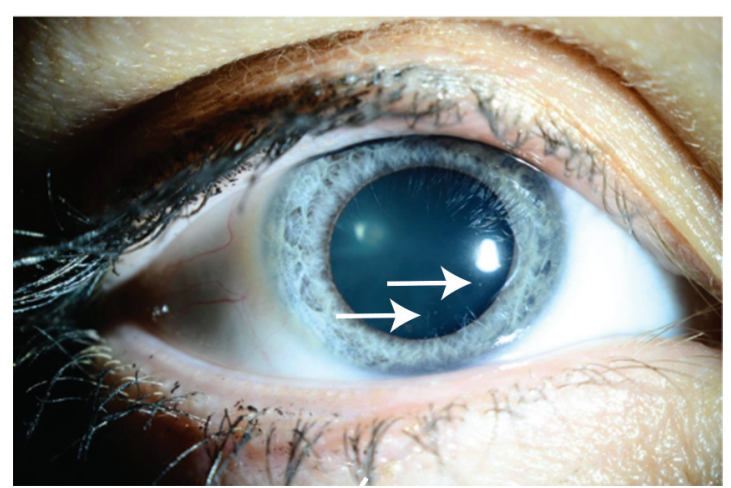

Fig 2 Uninflamed chronically painful white eye with panuveitis and reduced vision. Small keratic precipitates (inflammatory cell deposits on the corneal endothelium) are barely visible (arrows) 


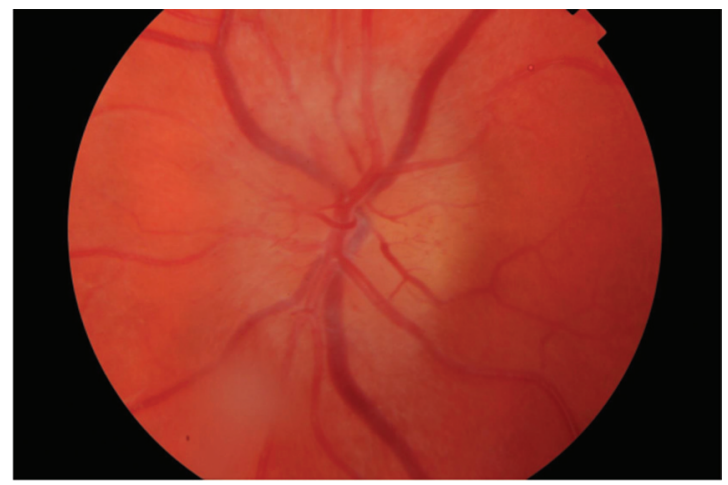

Fig 3 Funduscopy of an uninflamed, acutely painful (on movement) white eye showing the swollen disc of optic neuritis associated with acute loss of vision

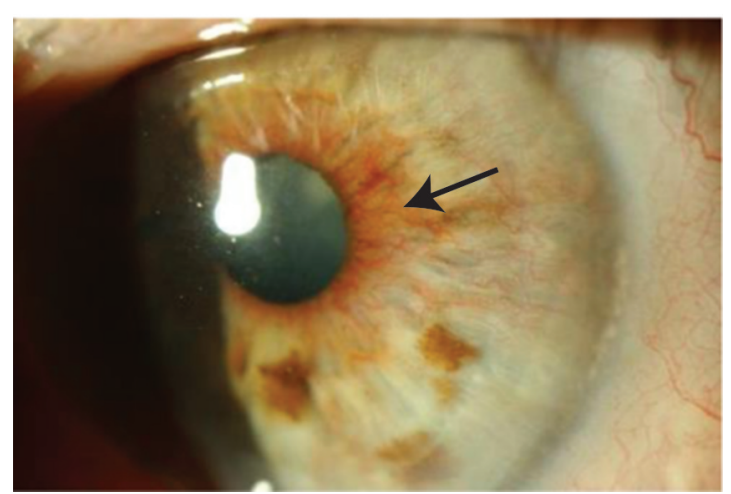

Fig 4 Uninflamed painful white eye showing fine abnormal "rubeotic" iris new vessels (arrow). Rubeosis of the iris is commonly associated with secondary glaucoma

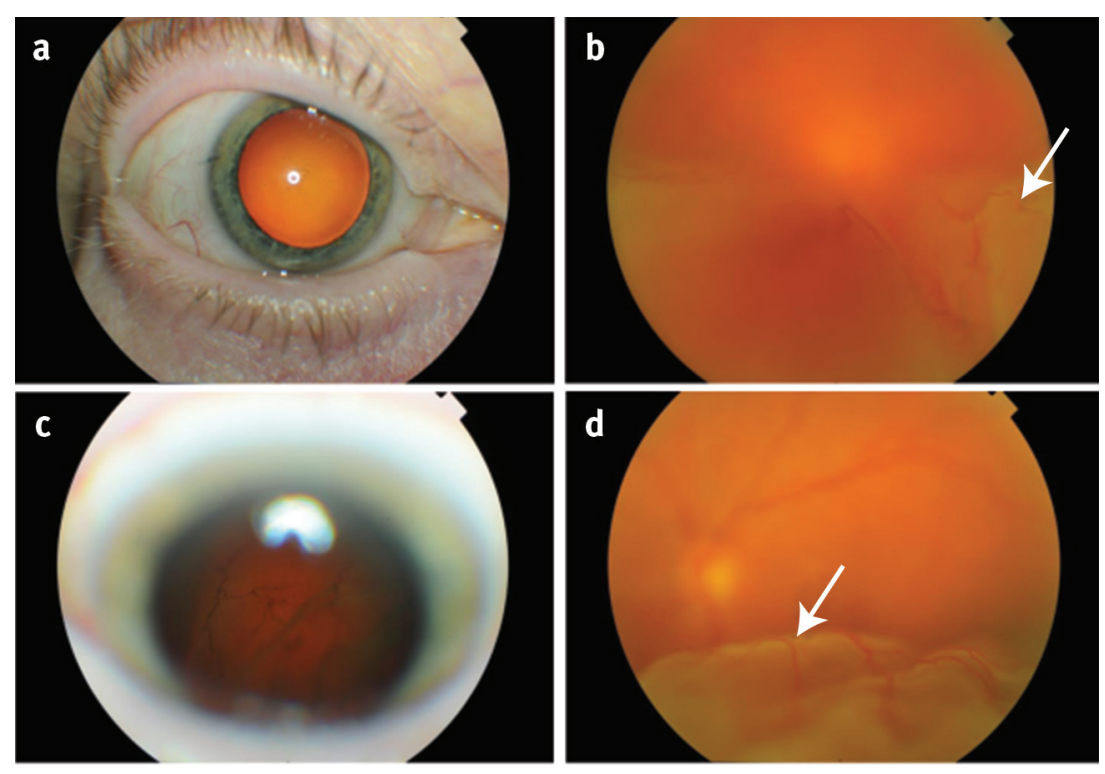

Fig 5 Uninflamed bilateral severely painful eyes due to posterior scleritis showing exudative retinal detachment and vitreous "haze" (infiltration with inflammatory cells and protein exudate). (a) external appearance of right white, uninflamed eye; (b) fundus appearance of right eye in (a) showing vitreous "haze" (indistinct view of optic disc and retinal vessels) and inferior retinal detachment (arrow); (c) and (d) same as (a) and (b) but left eye 


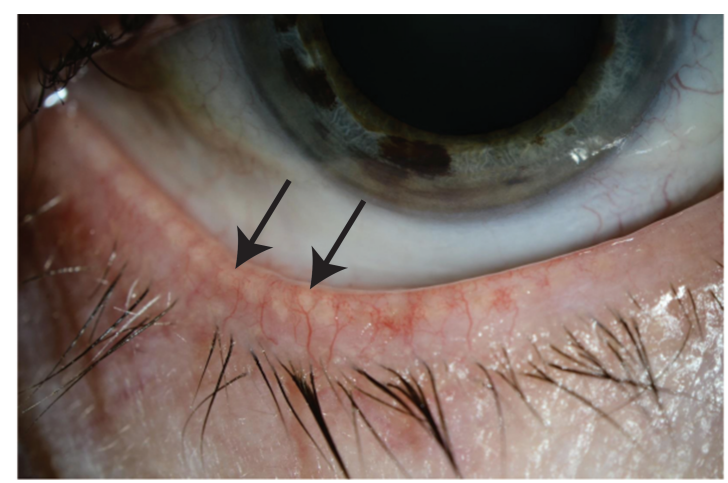

Fig 6 Uninflamed, mildly painful ("irritated") eye due to meibomitis, causing a disturbed tear film (lack of oily layer) and excessive tear evaporation, with secondary "dry eye" disease. The openings of the meibomian gland ducts are blocked as shown by the yellow spots on the lower lid (arrows) 